PROCEEDINGS OF THE

AMERICAN MATHEMATICAL SOCIETY

Volume 68, Number 1, January 1978

\title{
PRODUCTS OF SINGULAR CONTINUOUS MEASURES
}

\author{
ALAN MACLEAN
}

\begin{abstract}
Let $G$ be a compact Abelian group. It is shown that if the Fourier transform of $f \in A(G)$ satisfies certain lacunary conditions, then $f$ may be factored as the convolution product of singular continuous measures.
\end{abstract}

Let $G$ be a compact Abelian group with dual group $\Gamma$. Doss [3] has shown that if $\mu$ is any continuous measure on $G$, then there exists a singular measure $\nu \neq 0$ on $G$ such that $(\mu * \nu)^{\wedge} \in L^{1}(\Gamma)$, i.e., the Radon-Nikodým derivative of $\mu * \nu$ has an absolutely convergent Fourier series. Extensions of this theorem to locally compact groups are given in [4]. In this paper we prove a partial converse to Doss's theorem. We show (Theorem 1) that if $f$ has an absolutely convergent Fourier series and $\hat{f}$ satisfies certain lacunary conditions, then there are singular continuous measures $\mu$ and $\nu$ such that $f=\mu * \nu$ and $\|\mu\|$ $\|\nu\|<\|\hat{f}\|_{1}$. We then list some consequences of this theorem and give some examples.

Throughout $G$ will denote a compact Abelian group with dual $\Gamma ; M(G)$ the complex regular Borel measures on $G ; A(G)=\left\{f \in C(G): \hat{f} \in L^{1}(\Gamma)\right\}$ with $\|f\|_{A}=\|\hat{f}\|_{1}$. For $\mu \in M(G)$ we denote the support of $\hat{\mu}$ by $S(\hat{\mu})=\{\gamma \in \Gamma$ : $\hat{\mu}(\gamma) \neq 0\}$. We follow the exposition on dissociate sets and Riesz products given in [2]. In particular, $\Theta \subset \Gamma$ is dissociate if $0 \notin \Theta$ and each $\gamma \in \Gamma$ can be written in at most one way (except for order of summands) as

$$
\gamma=\sum_{1}^{n} \varepsilon_{j} \gamma_{j}
$$

where the $\gamma_{j}(1 \leqslant j \leqslant n)$ are distinct elements of $\Theta, \varepsilon_{j} \in\{ \pm 1\}$ if $2 \gamma_{j} \neq 0$, and $\varepsilon_{j}=1$ if $2 \gamma_{j}=0 . \Omega(\Theta)$ consists of 0 and all characters of the form (1). We will make use of the following: if $\mu$ is a Riesz product generated by $b, \Theta$ where, say, $|b(\theta)| \leqslant \frac{1}{2}, b(\theta) \neq 0$ for all $\theta \in \Theta$, then $\mu$ is singular if $\Sigma_{\theta}|b(\theta)|^{2}$ $=\infty$ and $\mu$ is continuous if $\Sigma_{\theta}(1-|b(\theta)|)=\infty$. These facts and much additional information may be found in [1], [2], [6], [8], and [10].

THEOREM 1. Let $f \in A(G)$ and suppose there exists an infinite dissociate set $\Theta \subset \Gamma$ such that $[S(\hat{f})-S(\hat{f})] \cap \Omega(\Theta)$ is finite. Then there exist singular continuous measures $\mu$ and $\nu$ on $G$ such that $f=\mu * \nu$ and $\|\mu\|\|\nu\| \leqslant\|f\|_{A}$.

Received by the editors April 19, 1977.

AMS (MOS) subject classifications (1970). Primary 43A25, 42 A96.

Key words and phrases. CA group, absolutely convergent Fourier series, convolution, singular continuous measure. 
Proof. By removing a finite set from $\Theta$ we may assume without loss of generality that

$$
[S(\hat{f})-S(\hat{f})] \cap \Omega(\Theta)=\{0\} .
$$

Write $\Theta=\Psi \cup \Lambda$ where both $\Psi$ and $\Lambda$ are infinite and $\Psi \cap \Lambda=\varnothing$. Choose an increasing sequence $\left(F_{n}\right)$ of finite subsets of $\Gamma$ such that $S(\hat{f})=\bigcup_{1}^{\infty} F_{n}$. Next write

$$
\hat{f}(\gamma)=|\hat{f}(\gamma)|^{1 / 2}|\hat{f}(\gamma)|^{1 / 2} \operatorname{sgn} \overline{\hat{f}}(\gamma)
$$

Then

$$
f=g * h \text { and }\|f\|_{A}=\|g\|_{2}\|h\|_{2}
$$

where $g, h \in L^{2}(G)$ are defined by $\hat{g}=|\hat{f}|^{1 / 2}$ and $\hat{h}=|\hat{f}|^{1 / 2} \operatorname{sgn}(\overline{\hat{f}})$.

Let $\sigma$ be any singular continuous Riesz product generated by $b, \Psi$ (e.g., $\left.b(\theta) \equiv \frac{1}{2}\right) . \sigma$ is a probability measure with $S(\hat{\sigma}) \subset \Omega(\Psi) \subset \Omega(\Theta)$. In particular, (1) implies that

$$
\hat{\boldsymbol{\sigma}}(\gamma-\chi)=0 \text { for all } \gamma, \chi \in S(\hat{f}), \gamma \neq \chi .
$$

Thus, setting $Q_{n}=\Sigma_{F_{n}} \hat{g}(\gamma) \gamma(n \geqslant 1)$ we have for $m \geqslant n$

$$
\int_{G}\left|Q_{m}-Q_{n}\right|^{2} d \sigma=\sum_{F_{m} / F_{n}} \hat{g}(\gamma) \overline{\hat{g}(\chi)} \hat{\sigma}(\chi-\gamma)=\sum_{F_{m} / F_{n}}|\hat{g}(\gamma)|^{2} .
$$

It follows that the sequence $\left(Q_{n}\right)$ is Cauchy in $L^{2}(\sigma)$. Let $g^{\prime}$ denote the $L^{2}$ limit of the $Q_{n}$ and let $\mu \in M(G)$ be the measure whose Radon-Nikodým derivative with respect to $\sigma$ is $g^{\prime}: \mu=g^{\prime} \sigma$. Then $\mu$ is singular and continuous since $\sigma$ is. Further, $\left(Q_{n}\right)$ converges weakly to $g^{\prime}$ in $L^{2}(\sigma)$ so that

$$
\hat{\mu}(\chi)=\lim _{n \rightarrow \infty} \sum_{\gamma \in F_{n}} \hat{g}(\gamma) \hat{\sigma}(\chi-\gamma) \quad(\chi \in \Gamma) .
$$

In particular, for $\chi \in S(\hat{f})$, (2) shows that $\hat{\mu}(\chi)=\hat{g}(\chi)$. Moreover,

$$
\|\mu\| \leqslant\left\|g^{\prime}\right\|_{2, \sigma} \leqslant\|\hat{g}\|_{2}=\|g\|_{2} \text {. }
$$

Next, let $\tau$ be any singular continuous Riesz product on $\Lambda$, set $P_{n}=$ $\Sigma_{F_{n}} \hat{h}(\gamma) \gamma$ and repeat the above argument to obtain a singular continuous measure $\nu=h^{\prime} \tau$ such that $\hat{\nu}=\hat{h}$ on $S(\hat{f})$ and $\|\mu\| \leqslant\|h\|_{2}$.

Suppose $(\mu * \nu)^{\wedge}(\chi) \neq 0$. It follows from (3) that $\hat{\sigma}(\chi-\gamma) \neq 0$ for some $\gamma \in S(\hat{f})$, whence, $\chi=\gamma+\omega$ for some $\gamma \in S(\hat{f}), \omega \in \Omega(\Psi)$. Similarly, $\chi=$ $\gamma^{\prime}+\omega^{\prime}$ for some $\gamma^{\prime} \in S(\hat{f})$ and $\omega^{\prime} \in \Omega(\Lambda)$. Now $\Psi \cap \Lambda=\varnothing$ and $\Theta$ is dissociate. This shows first that $\gamma-\gamma^{\prime}=\omega^{\prime}-\omega \in \Omega(\Theta)$ and then in combination with (1) implies that $\omega=\omega^{\prime}=0$, i.e., $\chi=\gamma=\gamma^{\prime} \in S(\hat{f})$. Thus, $(\mu * \nu)^{\wedge}$ vanishes off $S(\hat{f})$. On the other hand, from above $(\mu * \nu)^{\wedge}=$ $(g * h)^{\wedge}=\hat{f}$ on $S(\hat{f})$. Thus, $f=\mu * \nu$. Finally, $\|\mu\|\|\nu\| \leqslant\|g\|_{2}\|h\|_{2}=\|f\|_{A}$.

For $\mathscr{F} \subset A(G)$ and $E \subset \Gamma$, put $\mathscr{F}_{E}=\{f \in \mathscr{F}: S(\hat{f}) \subset E\}$. We say that $f \in \mathscr{F}_{E}$ admits singular continuous factorization if there exist singular continuous measures $\mu$ and $\nu$ such that $f=\mu * \nu$ and $\|\mu\|\|\nu\| \leqslant\|f\|_{A}$. 
CoRollary 1. If $E \subset \Gamma$ is a Sidon set such that $(E-E) \cap \Omega(\Theta)$ is finite for some infinite dissociate set $\Theta$, then each $f \in L_{E}^{\infty}(G)$ admits singular continuous factorization.

COROllary 2. Let $E \subset \Gamma$. If there exists a continuous measure $\sigma \in M(G)$ such that $|\hat{\sigma}| \geqslant \delta>0$ on $E-E$, then each $f \in A_{E}(G)$ admits singular continuous factorization.

Proof. Corollary 1 is clear. Corollary 2 follows from Lemma 1, Doss [3] (or Lemma 5, [4]): if $\sigma$ is continuous and $\delta>0$, then there exists an infinite dissociate set $\Theta$ such that $|\hat{\sigma}(\omega)|<\delta$ for all $\omega \in \Omega(\Theta) \backslash\{0\}$. Thus, $(E-E) \cap$ $\Omega(\Theta)=\{0\}$.

REMARKS. Every dissociate set $\Theta \subset \Gamma$ satisfies the hypothesis of Corollary 2: If $\mu$ is the continuous Riesz product with $\hat{\mu}(\theta)=\frac{1}{2}$ for every $\theta \in \Theta$, then $\hat{\mu}\left(\theta_{1}-\theta_{2}\right) \geqslant \frac{1}{4}$ for all $\theta_{1}, \theta_{2} \in \Theta$.

Every Sidon set $E$ satisfies $E \cap \Omega(\Theta)=\{0\}$ for some infinite dissociate set $\Theta$. For by the Hartman-Wells Theorem $[7,4.8]$ there is a continuous measure $\mu$ such that $\hat{\mu}=1$ on $E$, hence, the lemma of Doss cited above provides the required $\Theta$. Of course, this argument fails for $E-E$ since this set is not Sidon. Indeed, not every Sidon set satisfies the hypothesis of Corollary 1; $E=\left\{4^{n}, 4^{n}+n: n \geqslant 1\right\}$ is a Sidon set for which $E-E=Z$. On the other hand, there are rather "large" sets $E$ satisfying $(E-E) \cap \Omega(\Theta)=\{0\}$. For example, one can construct infinite dissociate sets $\Theta_{1}$ and $\Theta_{2}$ for which $\left[\Omega\left(\Theta_{1}\right)-\Omega\left(\Theta_{1}\right)\right] \cap \Omega\left(\Theta_{2}\right)=\{0\}$. Since $\Omega\left(\Theta_{1}\right)$ supports transforms of nonzero singular measures this shows, in particular, that there are non-Riesz sets $E$ ( $E$ is a Riesz set if $S(\hat{\mu}) \subset E$ implies $\mu \in M_{a}(G)$ ) for which every $f \in A_{E}(G)$ admits singular continuous factorization. It may be the case, in fact, that every $f \in A(G)$ (perhaps even $f \in L^{1}(G)$ ) admits such a factorization. Little appears to be known in this direction. It is known that not every continuous measure can be factored as a product of continuous measures [9].

Corollary 3. Let $H$ be an infinite closed subgroup of $G$. Then each $f \in A(G)$ that is constant on cosets of $H$ admits singular continuous factorization. In particular, each continuous positive definite function on $G$ for which $\{x \in G: f(x)=f(0)\}$ is infinite admits such a factorization.

Proof. $H$ is not discrete so Haar measure $m$ on $H$ may be regarded as a continuous measure in $M(G)$. Since $\hat{m}$ is the characteristic function of the annihilator $E=\{\gamma \in \Gamma: \gamma=1$ on $H\}$ of $H$, Corollary 2 shows that each $f \in A(G)$ with $S(\hat{f}) \subset E-E=E$ admits the asserted factorization. These functions are exactly those of the form $g \circ \pi$ where $g \in A(G / H)$ and $\pi$ : $G \rightarrow G / H$ is the quotient map, i.e., they are the members of $A(G)$ that are constant on cosets of $H$. Finally, if $f$ is continuous positive definite on $G$, then $H=\{x \in G: f(x)=f(0)\}$ is a closed subgroup upon whose cosets $f$ is constant.

For $G=T$ it is possible to construct a large collection of sets $E \subset Z$ for 
which each $f \in A_{E}$ admits singular continuous factorization. We begin with a definition and a lemma whose proof is straightforward and omitted.

A set $E \subset Z$ is said to have (arbitrarily) large symmetric gaps relative to $I_{n}$ if there exist intervals $I_{n}=\left[a_{n}, b_{n}\right]$ where $a_{n}, b_{n}$ are positive integers with $a_{n}<b_{n}<a_{n+1}$ such that $\lim _{n \rightarrow \infty}\left(b_{n}-a_{n}\right)=\infty$ and

$$
\liminf _{n \rightarrow \infty} n^{-1}\left|E \cap \bigcup_{1}^{n}\left(-I_{k} \cup I_{k}\right)\right|<1
$$

$(|A|=$ cardinality of $A)$. We note that this occurs, for instance, if there exists a function $g>0$ such that

$$
\liminf _{n \rightarrow \infty} n^{-1} g(n)=0
$$

and $\left|E \cap \cup_{1}^{n}\left(-I_{k} \cup I_{k}\right)\right| \leqslant \rho g(n)(n \geqslant 1)$ for some constant $\rho>0$. In particular, Sidon sets $(g(n)=\log n)$ and, more generally, $\Lambda_{p}$ sets $(p>2)$ $\left(g(n)=n^{2 / p}\right)$ have large symmetric gaps [5, 37.25].

LEMMA. Let $\left(x_{k}\right)_{1}^{\infty}$ be a sequence of positive integers with $x_{k} \geqslant 3$ for all $k>2$. Let $\theta_{n}=\prod_{1}^{n} x_{k}, \Theta=\left(\theta_{n}\right)_{1}^{\infty}$, and set

$$
\Omega_{n}=\left[\theta_{n}, \sum_{1}^{n} \theta_{k}\right] \cup\left[\theta_{n+1}-\sum_{1}^{n} \theta_{k}, \theta_{n+1}-1\right] \quad(n>1) .
$$

Then $\theta_{n+1}>2 \sum_{1}^{n} \theta_{k}(n \geqslant 1)$ so that $\Theta$ is dissociate and

$$
\Omega(\Theta) \subset\left(\bigcup_{1}^{\infty} \Omega_{n}\right) \cup\{0\} \cup\left(-\bigcup_{1}^{\infty} \Omega_{n}\right) \text {. }
$$

TheOReM 2. Let $E \subset Z$. If $E-E$ has large symmetric gaps relative to $I_{n}$, then each $f \in A_{E}(T)$ admits singular continuous factorization.

Proof. Let $I_{n}=\left[a_{n}, b_{n}\right]$ be as in the definition and for a sequence $\left(x_{n}\right)$ of integers set $\theta_{n}=\prod_{1}^{n} x_{k}, \Theta=\left(\theta_{n}\right)_{1}^{\infty}$. For $n \geqslant 1$, let

$$
F_{n}=\left[\theta_{n}-\sum_{1}^{n-1} \theta_{k}, \theta_{n}+\sum_{1}^{n-1} \theta_{k}\right]
$$

It suffices by Theorem 1 to show that there is an infinite dissociate set $\Theta$ such that $(E-E) \cap \Omega(\Theta)=\{0\}$. In view of the Lemma we need only show that a sequence $\left(x_{n}\right)$ can be chosen satisfying $x_{n} \geqslant 3(n \geqslant 1)$ and $(E-E) \cap \cup_{1}^{\infty}$ $\left(-F_{n} \cup F_{n}\right)=\varnothing$. We do this by induction. Let $x_{1} \geqslant 3$ be any integer such that $\pm x_{1} \notin E-E$. Then $(E-E) \cap\left\{ \pm x_{1}\right\}=(E-E) \cap\left(-F_{1} \cup F_{1}\right)=$ $\varnothing$. Assume $x_{k} \geqslant 3(1 \leqslant k \leqslant n)$ have been chosen so that $(E-E) \cap \cup_{1}^{n}$ $\left(-F_{k} \cup F_{k}\right)=\varnothing$. The induction will be complete provided $x_{n+1} \geqslant 3$ can be selected such that $(E-E) \cap\left(-F_{n+1} \cup F_{n+1}\right)=\varnothing$. Suppose to the contrary that for each $m \geqslant 3$,

$$
(E-E) \cap\left(-H_{m} \cup H_{m}\right) \neq \varnothing
$$

where 


$$
H_{m}=\left[\theta_{n} m-\sum_{1}^{n} \theta_{k}, \theta_{n} m+\sum_{1}^{n} \theta_{k}\right] .
$$

Since $a_{n}<a_{n+1}(n \geqslant 1)$ and $\lim _{n \rightarrow \infty}\left(b_{n}-a_{n}\right)=\infty$ there is a $N$ such that $\theta_{n}^{-1}\left(a_{j}+\sum_{1}^{n} \theta_{k}\right) \geqslant 3$ and $b_{j}-a_{j}>\theta_{n}+2 \sum_{1}^{n} \theta_{k}$ for all $j>N$. Then

$$
\theta_{n}^{-1}\left(b_{j}-\sum_{1}^{n} \theta_{k}\right)-\theta_{n}^{-1}\left(a_{j}+\sum_{1}^{n} \theta_{k}\right)>1 .
$$

Hence, for each $j \geqslant N$ there is an integer $m_{j} \geqslant 3$ satisfying

$$
\theta_{n}^{-1}\left(a_{j}+\sum_{1}^{n} \theta_{k}\right) \leqslant m_{j} \leqslant \theta_{n}^{-1}\left(b_{j}-\sum_{1}^{n} \theta_{k}\right) \text {. }
$$

It follows that

$$
H_{m_{j}}=\left[\theta_{n} m_{j}-\sum_{1}^{n} \theta_{k}, \theta_{n} m_{j}+\sum_{1}^{n} \theta_{k}\right] \subset\left[a_{j}, b_{j}\right] .
$$

Further, from (2)

$$
\theta_{n} m_{j}+\sum_{1}^{n} \theta_{k} \leqslant b_{j}<a_{j+1} \leqslant \theta_{n} m_{j+1}-\sum_{1}^{n} \theta_{k}
$$

so that the intervals $H_{m_{j}}$ in (3) are pairwise disjoint. Thus, the assumption in (1) leads to

$$
\begin{aligned}
M-N+1 & \leqslant\left|(E-E) \cap \bigcup_{j=N}^{M}\left(-H_{m_{j}} \cup H_{m_{j}}\right)\right| \\
& \leqslant\left|(E-E) \cap \bigcup_{j=1}^{M}\left(-I_{j} \cup I_{j}\right)\right|
\end{aligned}
$$

for all $M \geqslant N$. This contradicts line (*) above and completes the proof.

\section{REFERENCES}

1. G. Brown, Riesz products and generalized characters, Proc. London Math. Soc. (3) 30 (1975), 209-238.

2. G. Brown and W. Moran, On orthogonality of Riesz products, Proc. Cambridge Philos. Soc. 76 (1974), 173-181.

3. R. Doss, Convolution of singular measures, Studia Math. 45 (1973), 111-117.

4. C. C. Graham and A. MacLean, A multiplier theorem for continuous measures, Studia Math. (to appear).

5. E. Hewitt and K. A. Ross, Abstract harmonic analysis, Volumes I and II, Springer-Verlag, New York-Heidelberg-Berlin, 1963 and 1970.

6. E. Hewitt and H. S. Zuckerman, Singular measures with absolutely continuous convolution squares, Proc. Cambridge Philos. Soc. 62 (1966), 399-420. Corrigendum, ibid. 63 (1967), 367-368.

7. J. López and K. A. Ross, Sidon sets, Marcel Dekker, New York, 1975.

8. O. Padé, Sur le spectre d'une classe de produits de Riesz, C. R. Acad. Sci. Paris Sér. A-B 276 (1973), A1453-A1455.

9. D. L. Salinger and N. Th. Varopoulus, Convolutions of measures and sets of analyticity, Math. Scand. 25 (1969), 5-18.

10. A. Zygmund, On lacunary trigonometric series, Trans. Amer Math. Soc. 34 (1932), 435-446.

Department of Mathematics, Wichita State University, Wichita, Kansas 67208 